Pacific Journal of Mathematics

SUBNORMAL OPERATORS IN STRICTLY CYCLIC OPERATOR 


\title{
SUBNORMAL OPERATORS IN STRICTLY CYCLIC OPERATOR ALGEBRAS
}

\author{
RICHARD BOLSTEIN AND WARREN WOGEN
}

\begin{abstract}
It is shown that a subnormal operator cannot belong to a strictly cyclic and separated operator algebra unless it is normal and has finite spectrum. Further, a subnormal operator not of this type cannot have a strictly cyclic commutant.
\end{abstract}

1. Let $\mathscr{H}$ be a complex Hilbert space, and let $\mathscr{A}$ be a subset of the algebra $\mathscr{B}(\mathscr{H})$ of all bounded linear operators on $\mathscr{H}$. A vector $x \in \mathscr{H}$ with the property that $\mathscr{A} x=\{A x: A \in \mathscr{A}\}$ is the full Hilbert space is said to be a strictly cyclic vector for $\mathscr{A}$, and $\mathscr{A}$ is said to be strictly cyclic if such a vector exists. A vector $x$ is called a separating vector for $\mathscr{A}$ if no two distinct operators in $\mathscr{A}$ agree at $x$. The set $\mathscr{A}$ is said to be strictly cyclic and separated if there is a vector $x$ which is both strictly cyclic and separating for $\mathscr{A}$.

Strictly cyclic operator algebras have recently been investigated by Mary Embry [2] and Alan Lambert [3]. Let $\mathscr{A}^{\prime}$ denote the commutant of the set $\mathscr{A}$, that is, $\mathscr{A}^{\prime}$ is the set of all bounded linear operators which commute with every operator in $\mathscr{A}$. Note that if $x$ is a cyclic vector for $\mathscr{A}$ (meaning $\mathscr{A} x$ is dense in $\mathscr{H}$ ), then $x$ is separating for $\mathscr{A}^{\prime}$.

LEMMA 1. Let $\mathscr{A}$ be a strictly cyclic subset of $\mathscr{B}(\mathscr{H})$. If $\mathscr{A}$ is abelian, then it is maximal abelian, $\mathscr{A}=\mathscr{A}^{\prime}$. Thus, a strictly cyclic abelian subset is automatically a weakly closed algebra.

This lemma, which indicates the severity of the condition of strict cyclicity, is a sharper form of a result of Lambert [3].

Proof. Let $x$ be strictly cyclic for $\mathscr{A}$, and let $B \in \mathscr{A}^{\prime}$. Then there exists $A \in \mathscr{A}$ such that $A x=B x$. But $\mathscr{A} \subset \mathscr{A}^{\prime}$ by hypothesis, so $A \in \mathscr{A}^{\prime}$. Since $x$ is separating for $\mathscr{A}^{\prime}$, we have $B=A \in \mathscr{A}$, and the proof is complete.

If $\mathscr{A}$ is strictly cyclic and abelian, then it is strictly cyclic and separated by Lemma 1. Mary Embry [2] showed that the converse holds if $\mathscr{A}$ is the commutant of a single operator. Thus, if $A$ is normal and $\{A\}^{\prime}$ is strictly cyclic and separated, then $\{A\}^{\prime}$ consists of normal operators by Fuglede's theorem. In a private communication to the authors, Mary Embry asked if "normal" could be replaced by "subnormal" in this statement. An operator is called subnormal if 
it is the restriction of a normal operator to an invariant subspace. To this end, we show that if $A$ is subnormal then strict cyclicity of $\{A\}^{\prime}$ already forces $A$ to be normal, and, moreover, its spectrum is a finite set. Thus, the commutant of a subnormal operator cannot be strictly cyclic and separated unless the underlying Hilbert space is finite-dimensional (since the commutant is then abelian and hence the operator, which is normal, must have simple spectrum). More generally, it is shown that a uniformly closed subalgebra $\mathscr{A}$ of $\mathscr{B}(\mathscr{C})$ which has a separating vector $x$ with the property that $\mathscr{A} x$ is a closed subspace of $\mathscr{H}$ (this is the case if $x$ is also strictly cyclic) contains no subnormal operators except possibly for normal operators with finite spectrum.

2. Let $\mu$ be a finite positive Borel measure in the plane with compact support $X$, let $H^{2}(\mu)$ be the closure of the polynomials in $L^{2}(\mu)$, and put $H^{\infty}(\mu)=H^{2}(\mu) \cap L^{\infty}(\mu)$. The next theorem, which is used to derive the main result, may be of independent interest.

\section{THEOREM 1. $H^{\infty}(\mu)=H^{2}(\mu)$ if, and only if, $X$ is finite.}

Proof. The sufficiency is trivial. Assume now that $X$ is infinite. Note that the inclusion map of $H^{\infty}(\mu)$ into $H^{2}(\mu)$ is continuous. We will show that the inverse map is not continuous, and hence, by the Open Mapping Theorem, that $H^{\infty}(\mu) \neq H^{2}(\mu)$.

Since $X$ is compact and infinite, its set $X^{\prime}$ of accumulation points is compact and nonempty. Choose $\lambda_{0} \in X^{\prime}$ such that $\left|\lambda_{0}\right|=\max \{|\lambda|$ : $\left.\lambda \in X^{\prime}\right\}$, and let $D_{1}=\left\{\lambda:|\lambda| \leqq\left|\lambda_{0}\right|\right\}$. By the choice of $\lambda, X \backslash D_{1}$ is a countable set. Therefore, we can choose a closed disk $D_{2}$ which contains $D_{1}$ and is tangent to $D_{1}$ at $\lambda_{0}$, in such a way that the boundary of $D_{2}$ intersects $X$ only at $\lambda_{0}$. Now note that we may as well assume that $D_{2}$ is the closed unit disc $\Delta$, and that $\lambda_{0}=1$.

Now $X \backslash 4$ is a countable set $\left\{y_{1}, y_{2}, \cdots\right\}$, and if this set infinite, we must have $\lim y_{n}=1$. Let $K=\Delta \cup(X \backslash \Delta)$. Then $K$ is a compact set which does not separate the plane. Define a sequence of functions $\left\{f_{n}\right\}$ on $K$ by

$$
f_{n}(z)=\left\{\begin{array}{l}
z^{n}: z \in \Delta \\
0: \quad z=y_{i}, 1 \leqq i \leqq n \\
1: \quad z=y_{i}, i>n .
\end{array}\right.
$$

Then, for each $n, f_{n}$ is continuous on $K$ and analytic in its interior. By Mergelyan's theorem, each $f_{n}$ is the uniform limit on $K$ of a sequence of polynomials. Hence each $f_{n} \in H^{\infty}(\mu)$.

Let $\chi$ denote the function which has the value 1 at the point 1 
and the value zero elsewhere. Clearly, $f_{n} \rightarrow \chi$ pointwise, and hence in the metric of $L^{2}(\mu)$ by dominated convergence. In particular, $\chi \in H^{\infty}(\mu)$. However, the point 1 is an accumulation point of the support of $\mu$, and hence $\left\|f_{n}-\chi\right\|_{\infty}=1$ for every $n$. Thus, $\left\{f_{n}\right\}$ converges to $\chi$ in $H^{2}(\mu)$ but not in $H^{\infty}(\mu)$.

THEOREM 2. Let $S$ be a subnormal operator on the Hilbert space $\mathscr{H}$, let $\mathscr{A}$ be the uniformly closed algebra generated by $S$. If $\mathscr{A}$ has a separating vector $x$ such that $\mathscr{A} x$ is a closed subspace of $\mathscr{H}$, then the spectrum of $S$ is a finite set, and hence $S$ is normal.

Proof. Let $\mathscr{B}$ be the uniformly closed algebra generated by $S$ and the identity operator $I$. Since $\mathscr{B} x$ is the sum of $\mathscr{A} x$ and the one-dimensional space spanned by $x$, and since we assume that $\mathscr{A} x$ is closed, we also have that $\mathscr{B} x$ is a closed subspace of $\mathscr{H}$.

Now $\mathscr{B} x$ is invariant under $S$ and the restriction operator $S_{0}=S \mid \mathscr{B} x$ is subnormal. Since the uniformly closed algebra $\mathscr{B}_{0}$ generated by $S_{0}$ and $I$ contains $\mathscr{B} \mid \mathscr{B} x$, it follows that $x$ is a strictly cyclic vector for $\mathscr{B}_{0}$, that is, $\mathscr{B}_{0} x=\mathscr{B} x$. By the representation theorem for subnormal operators with a cyclic vector, Bram [1], $S_{0}$ is unitarily equivalent to the operator of multiplication by the identity function on some $H^{2}(\mu)$ space. Furthermore, the unitary equivalence can be constructed so that $x$ corresponds to the constant function 1 .

Now $\mathscr{B}_{0}$ corresponds via the unitary equivalence to the algebra of multiplication operators $M_{\phi}: f \rightarrow \phi f$ on $H^{2}(\mu)$, where $\phi$ belongs to the $L^{\infty}(\mu)$-closure of the polynomials. Since any such function $\phi$ belongs to $H^{\infty}(\mu)$, it follows that the constant function 1 is a strictly cyclic vector for $\left\{M_{\phi}: \phi \in H^{\infty}(\mu)\right\}$, and hence that $H^{\infty}(\mu)=H^{2}(\mu)$. By Theoorem $1, H^{2}(\mu)$ is finite-dimensional.

It follows that $\mathscr{B} x$ is finite-dimensional, and, since $\mathscr{A} \subset \mathscr{S}$, so is $\mathscr{A} x$. Since $x$ separates $\mathscr{A}$, it follows that $\mathscr{A}$ is finite-dimensional. So there is a polynomial $p$ such that $p(S)=0$. Since $p(\sigma(S))=\sigma(p(S)$ ) $=\{0\}, \sigma(S)$ in finite and hence $S$ is normal.

Conollary 1. Let $\mathscr{A}$ be a uniformly closed subalgebra of $\mathscr{B}(\mathscr{H})$ which has a separating vector $x$ such that $\mathscr{A} x$ is a closed subspace of $\mathscr{H}$. (This is the case if $\mathscr{A}$ is strictly cyclic and separated.) Then $\mathscr{A}$ contains no subnormal operator with infinite spectrum.

Proof. Suppose $S \in \mathscr{A}$ is subnormal, and let $\mathscr{A}(S)$ be the uniformly closed algebra generated by $S$. Since $\mathscr{A}(S) \subset \mathscr{A}, x$ separates $\mathscr{A}(S)$. Since the linear transformation $A \rightarrow A x$ of $\mathscr{A}$ onto $\mathscr{A} x$ is continuous and one-to-one, and since $\mathscr{A} x$ is closed by hypothesis, the transformation has a continuous inverse by the Open Mapping Theorem. 
Therefore, $\mathscr{A}(S) x$ is closed, and the result follows from Theorem 2.

CoROLlaRY 2. The commutant of a subnormal operator $S$ is strictly cyclic if, and only if, $S$ is normal and has finite spectrum.

Proof. Suppose $\{S\}^{\prime}$ has a strictly cyclic vector $x$. Then $x$ separates $\{S\}^{\prime \prime}$, and it follows from [2, Lemma 2.1 (i)] that $\{S\}^{\prime \prime} x$ is a closed subspace. Thus, by Corollary 1, $S$ has finite spectrum and hence is normal.

Conversely, if $\sigma(S)=\left\{\lambda_{1}, \cdots, \lambda_{n}\right\}$, then each $\lambda_{j}$ is an eigenvalue and $\mathscr{H}$ is the direct sum of the corresponding eigensubspaces $\mathscr{H}_{j}$. It follows that $\{S\}^{\prime}=\mathscr{B}\left(\mathscr{H}_{1}\right) \oplus \cdots \oplus \mathscr{B}\left(\mathscr{H}_{n}\right)$. Hence any vector $x=x_{1}+\cdots+x_{n}$ where $0 \neq x_{j} \in \mathscr{H}_{j}, j=1, \cdots, n$, is strictly cyclic for $\{S\}^{\prime}$.

CoROLlaRY 3. Let $S$ be a subnormal operator on a Hilbert space $\mathscr{H}$. If $\{S\}^{\prime}$ is strictly cyclic and separated, then $\mathscr{H}$ is finite-dimensional.

Proof. By Corollary 2, $S$ is normal, its spectrum is finite, and $\{S\}^{\prime}=\mathscr{B}\left(\mathscr{H}_{1}\right) \oplus \cdots \oplus \mathscr{B}\left(\mathscr{H}_{n}\right)$ with notation as in the proof of that corollary. If $x$ is strictly cyclic for $\{S\}^{\prime}$, then $x=x_{1}+\cdots+x_{n}$ where $0 \neq x_{j} \in \mathscr{H}_{j}$, all $j$. If some $\mathscr{H}_{j}$ has dimension greater than 1 , then there is a nonzero operator $B_{j}$ on $\mathscr{C}_{j}$ which annihilates $x_{j}$, and hence there is a nonzero $B \in\{S\}^{\prime}$ such that $B x=0$. Therefore, if $\{S\}^{\prime}$ is strictly cyclic and separated, each $\mathscr{H}_{j}$ is one-dimensional and hence $\mathscr{H}=\mathscr{H}_{1} \oplus \cdots \oplus \mathscr{H}_{n}$ is finite-dimensional.

CoRollary 4. Let $S$ be a subnormal operator on a Hilbert space $\mathscr{H}$. If $\{S\}^{\prime \prime}$ is strictly cyclic, then $\mathscr{H}$ is finite-dimensional.

Proof. If $x$ is strictly cyclic for $\{S\}^{\prime \prime} \subset\{S\}^{\prime}$, then it is strictly cyclic and separating for $\{S\}^{\prime}$ and the result follows from Corollary 3.

An operator $A$ is said to be strictly cyclic if the weakly closed algebra generated by $A$ and $I$ has this property. Since this algebra is contained in the second commutant of $A$, it follows that the second commutant of a strictly cyclic operator is strictly cyclic. In view of Corollary 4, we have

COROLLARY 5. There exist no strictly cyclic subnormal operators on an infinite-dimensional Hilbert space. 


\section{REFERENCES}

1. J. Bram, Subnormal operators, Duke Math. J., 22 (1955), 75-94.

2. Mary R. Embry, Strictly cyclic operator algebras on a Banach space, Pacific J. Math., 45 (1973),

3. Alan Lambert, Strictly cyclic operator algebras, Pacific J. Math., 39 (1971), 717727.

Received July 7, 1972.

George Mason University

AND

University of North Carolina at Chapel Hill 



\section{PACIFIC JOURNAL OF MATHEMATICS}

\section{EDITORS}

RICHARD ARENS (Managing Editor) University of California

Los Angeles, California 90024

R. A. Beaumon'T

University of Washington Seattle, Washington 98105
J. Dugundj1*

Department of Mathematics University of Southern California Los Angeles, California 90007

D. Gilbarg and J. Milgram Stanford University Stanford, California 94305

\section{ASSOCIATE EDITORS}

E. F. BECKENBACH

B. H. NeUMANN

F. WOLF

K. YOSHIDA

\section{SUPPORTING INSTITUTIONS}

UNIVERSITY OF BRITISH COLUMBIA CALIFORNIA INSTITUTE OF TECHNOLOGY UNIVERSITY OF CALIFORNIA MONTANA STATE UNIVERSITY UNIVERSITY OF NEVADA NEW MEXICO STATE UNIVERSITY OREGON STATE UNIVERSITY UNIVERSITY OF OREGON OSAKA UNIVERSITY

\section{UNIVERSITY OF SOUTHERN CALIFORNIA STANFORD UNIVERSITY UNIVERSITY OF TOKYO UNIVERSITY OF UTAH WASHINGTON STATE UNIVERSITY UNIVERSITY OF WASHINGTON AMERICAN MATHEMATICAL SOCIETY NAVAL WEAPONS CENTER}

* C. R. DePrima California Institute of Technology, Pasadena, CA 91109, will replace J. Dugundji until August 1974. 


\section{Pacific Journal of Mathematics}

\section{Vol. 49, No. $1 \quad$ May, 1973}

A. Bigard, Free lattice-ordered modules ...........................

Richard Bolstein and Warren R. Wogen, Subnormal operators in strictly cyclic

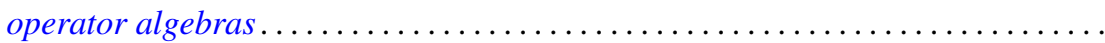

Herbert Busemann and Donald E. Glassco, II, Irreducible sums of simple

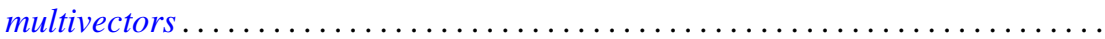

W. Wistar (William) Comfort and Victor Harold Saks, Countably compact groups

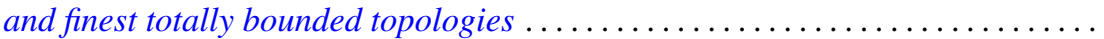

Mary Rodriguez Embry, Maximal invariant subspaces of strictly cyclic operator

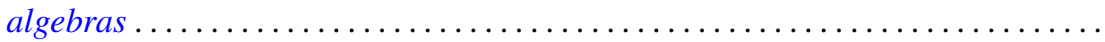

Ralph S. Freese and James Bryant Nation, Congruence lattices of semilattices......

Ervin Fried and George Grätzer, A nonassociative extension of the class of

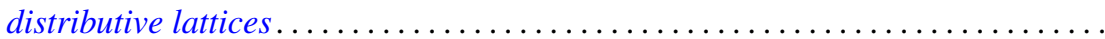

John R. Giles and Donald Otto Koehler, On numerical ranges of elements of locally

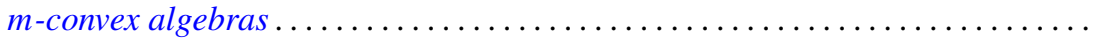

David A. Hill, On dominant and codominant dimension of $\mathrm{QF}-3$ rings ........ John Sollion Hsia and Robert Paul Johnson, Round and Pfister forms over $R(t) \ldots$ I. Martin (Irving) Isaacs, Equally partitioned groups . . . . . . . . . . . . . .

Athanassios G. Kartsatos and Edward Barry Saff, Hyperpolynomial approximation of solutions of nonlinear integro-differential equations.

Shin'ichi Kinoshita, On elementary ideals of $\theta$-curves in the 3-sphere and 2-links in the 4-sphere

Ronald Brian Kirk, Convergence of Baire measures

R. J. Knill, The Seifert and Van Kampen theorem via regular covering spaces ..

Amos A. Kovacs, Homomorphisms of matrix rings into matrix rings ..

Young K. Kwon, HD-minimal but no $H D$-minimal ..........

Makoto Maejima, On the renewal function when some of the mean renewal lifetimes are infinite

Juan José Martínez, Cohomological dimension of discrete modules over profinite groups.

W. K. Nicholson, Semiperfect rings with abelian group of units

Louis Jackson Ratliff, Jr., Three theorems on imbedded prime divisors of principal ideals.

Billy E. Rhoades and Albert Wilansky, Some commutants in $B(c)$ which are almost matrices

John Philip Riley Jr., Cross-sections of decompositions . . .

Keith Duncan Stroyan, A characterization of the Mackey uniformity $m\left(L^{\infty}, L^{1}\right)$ for

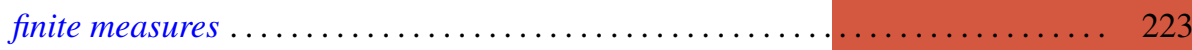

Edward G. Thurber, The Scholz-Brauer problem on addition chains . . . . . . . . . 229

Joze Vrabec, Submanifolds of acyclic 3-manifolds ............

Philip William Walker, Adjoint boundary value problems for compactified singular

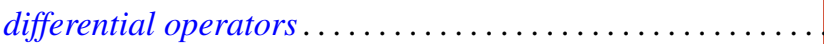

